\title{
CHEMICAL ABUNDANCE VERIFICATION OF CANDIDATE $\omega$ CENTAURI TIDAL DEBRIS STARS
}

\author{
JOY SKIPPER ${ }^{1}$
}

${ }^{1}$ University of Virginia

\begin{abstract}
The $\omega$ Centauri star system has several peculiar features that set it apart from other Milky Way globular clusters, such as its large mass, extended size, oblate shape, internal rotation, large age and metallicity spreads, and retrograde orbit. Because of these properties it is thought that $\omega$ Cen may instead be a heavily stripped remnant of a Milky Way-captured dwarf spheroidal galaxy, now currently orbiting (backwards) near the Galactic plane (e.g., Lee et al. 1999, Majewski et al. 2000). In an earlier study, high resolution spectroscopy was undertaken of a dozen $\omega$ Cen candidates identified by Majewski et al. (2012) on the basis of their $\omega$ Cen-like kinematics, and most were found by Majewski et al. to exhibit very high $[\mathrm{Ba} / \mathrm{Fe}]$ abundance patterns a characteristic currently thought to be uniquely characteristic of $\omega$ Cen stars with $[\mathrm{Fe} / \mathrm{H}] \gtrsim-1.7$. We expand on this previous work with an expanded subsample of stars chosen from the same parent sample. We compare derived $[\mathrm{Ba} / \mathrm{Fe}]$ abundances for these suspected stripped $\omega$ Cen stars to those stars in the $\omega$ Cen core, other globular cluster and field stars. For these data, we have employed multiple atomic transitions to derive reliable $[\mathrm{Ba} / \mathrm{Fe}]$ ratios. Of the 15 candidates observed, 12 exhibit barium abundances consistent with that of the $\omega$ Cen system, while the remaining 3 appear to be more consistent with typical Milky Way halo stars. These newly-confirmed $\omega$ Cen debris stars double the sample from the previous study and allows for a more complete mapping of $\omega$ Cen tidal debris and sheds further light upon the parent object's complex chemical and dynamical evolution. This provides strong evidence that a large majority of these retrograde stars in the $\omega$ Cen kinematical regime predicted by the model in Majewski et al. (2012) were indeed once a part of the $\omega$ Cen system.
\end{abstract}




\begin{tabular}{cccc}
\hline \hline & $\omega$ Centauri & Globular clusters & Dwarf galaxies \\
\hline Mass $\left(\times 10^{6} M_{\odot}\right)$ & 2.5 & 0.1 & 100 \\
Luminosity $\left(M_{V}\right)$ & -10 & -8 & -8 to -13 \\
Half-Light Radius $(\mathrm{pc})$ & 6 & 2 & $10-100$ \\
Global $M / L_{V}$ & 3 & 2 & 10 \\
Central $\sigma(\mathrm{km} / \mathrm{s})$ & 23 & 10 & $>10$ \\
Dark Matter & $\times$ & $\times$ & $\checkmark$ \\
$\Delta[\mathrm{Fe} / \mathrm{H}](\mathrm{dex})$ & 1 & 0 & $0.1-1.5$ \\
$\Delta$ Age $(\mathrm{Gyr})$ & 3 & 0 & $>1$ \\
Galactocentric Distance $(\mathrm{kpc})$ & 6 & $2-100$ & $>100$ \\
Central Surface Brightness $\left(V / \prime^{2}\right)$ & 16.7 & $15-20($ Harris 2010$)$ & 25 \\
$\log \left(T_{r_{h}}\right)(\mathrm{Gyr})$ & 10 & 8 to 9 & $>10$ \\
\hline
\end{tabular}

Table 1. Table 1.1 taken from Jalali (2011) which shows the parameters of $\omega$ Cen compared to other globular clusters and dwarf galaxies. All parameters for $\omega$ Centauri and GCs come from Harris (1996), except age and metallicity spread for $\omega$ Centauri from Hilker et al. (2004), and its mass from de Ven et al. (2006). Global $M / L_{v}$ and central $\sigma$ for globular clusters and $\omega$ Centauri from Pryor $\&$ Meylan (1993). Most of the parameters for dwarf galaxies are from Mateo (1998). The central brightness, luminosity, and effective radius values are from Bender et al. (1992).

\section{INTRODUCTION}

The currently accepted paradigm for the growth and evolution of large galaxies like the Milky Way is that this occurs through the hierarchical build up from smaller to larger systems. In the Milky Way today we see numerous examples of this process in the form of stellar streams of stars that have been stripped from smaller dwarf galaxies during "minor mergers." The most vivid example is that of the Sagittarius (Sgr) system Fellhauer et al. (2006) for which not only the dramatic tidal stream stars can be seen, but also the core, which has been in the process of tidal disruption for at least 3 billion years (Law \& Majewski (2016)). While globular clusters, like Pal 5 Odenkirchen et al. (2002), NGC 5466 Fellhauer et al. (2007) have also provided examples of currently ongoing tidal disruption of a star system, and evidence for such disruption has been suggested by several other dwarf spheroidal satellites of the Milky Way - e.g. Carina (Ricardo et al. (2008)) and Leo I Sohn et al. (2007) - on the basis of dwarf spheroidal stars seemingly just outside the dwarf spheroidal tidal radius, to date the Sgr system is the only Milky Way satellite galaxy for which widely-separated tidal debris has been definitively identified. This is unfortunate, as it would be much more illuminating to be able to explore a wider set of examples of this critical aspect of galaxy evolution.

However, there has long been speculation that the supposed globular cluster $\omega$ Centauri is actually a tidally stripped dwarf galaxy, due to its many characteristics unlike that of a typical globular cluster. The peculiarity of $\omega$ Centauri can be seen more quantitatively through the properties summarized in Table 1.1 from Jalali (2011), presented here as Table 1. $\omega$ Centauri is both much more massive than typical globular clusters and much less massive than typical dwarf galaxies. It's half-light radius, the radius that contains half of the light coming from the cluster, is also halfway between that of a globular cluster and that of a dwarf galaxy. If $\omega$ Centauri were indeed a stripped dwarf spheroidal, it would make sense that the mass and half-light radius would be between the definitions of globular clusters and dwarf galaxies, as many stars may have been stripped from a former " $\omega$ Centauri dwarf galaxy." The iron abundance and the age ranges of $\omega$ Cen may both fit easily within the ranges seen for dwarf galaxies, but don't match the single epoch, single metallicity trends of globular clusters. The iron abundance and age would be consistent with a dwarf galaxy, as the composition would remain like that of a dwarf galaxy during stripping. Finally, $\omega$ Centauri is moving in a retrograde orbit around the Milky Way, a peculiarity on its own that could be explained if $\omega$ Cen were the remains of a dwarf galaxy that happened to have merged into the Milky Way in a retrograde direction. Additionally, a retrograde disk orbit would be very destructive, and lead to more active stripping of stars from the dwarf galaxy, thus contributing more retrograde stars to the Milky Way orbit.

\begin{tabular}{ccccc} 
GGSS ID & RA (J2000) & Dec $(\mathrm{J} 2000)$ & 1 & $\mathrm{~b}$ \\
\hline \hline G1488-28.133 & 222.786117 & -28.241625 & 332.672 & 27.628 \\
G2109-61.187 & 318.586936 & -61.721363 & 333.252 & -40.374 \\
G2237-16.2017 & 339.92441 & -16.596104 & 45.122 & -57.788 \\
G1403+05.80 & 211.642118 & 5.211275 & 345.501 & 61.706 \\
G1532-05.29 & 233.616256 & -5.88037 & 359.131 & 38.601 \\
G1448-05.103 & 222.884405 & -6.001153 & 348.629 & 45.944 \\
G1527+22.523 & 232.512736 & 22.728033 & 34.882 & 53.919 \\
G1358-16.167 & 210.255848 & -17.006641 & 325.845 & 42.748 \\
G1443-16.11 & 221.470637 & -17.051704 & 338.354 & 37.825 \\
G1955-22.448 & 299.767554 & -22.53215 & 19.038 & -24.446 \\
G2215-39.109 & 334.727909 & -39.254696 & 2.097 & -56.162 \\
G1934-50.224 & 294.508007 & -50.41613 & 347.787 & -27.77 \\
G2222-45.34 & 336.684976 & -44.845295 & 351.710 & -56.1940 \\
G1851-39.1203 & 283.930745 & -39.355267 & 357.122 & -17.579 \\
G1942-44.358 & 296.571077 & -44.918907 & 354.193 & -28.122
\end{tabular}

Table 2: Current Study Star Information - Part 1

These peculiarities and the possibility of $\omega$ Cen being a stripped dwarf spheroidal nucleus has prompted searches for and chemical confirmation of stars that were once a part of the $\omega$ Cen system, but that would have been stripped away to leave the current state of $\omega$ Cen. This would constrain how $\omega$ Cen would have been stripped if this was the case. Mizutani et al. (2003) created a model predicting how this theoretical dwarf spheroidal, with what is now $\omega$ Cen as the core, would have stripped, showed that it accounted for two separate retrograde streams that exist in the Milky Way. Additionally, 


\begin{tabular}{cccccc} 
GGSS ID & $\mathrm{J}$ & $\mathrm{H}$ & $\mathrm{K}$ & $v_{G S R}$ & Reddening \\
\hline \hline G1488-28.133 & 8.305 & 7.664 & 7.501 & 112.82 & 0.144 \\
G2109-61.187 & 10.816 & 10.213 & 10.125 & 122.83 & 0.041 \\
G2237-16.2017 & 10.023 & 9.349 & 9.212 & -148.16 & 0.044 \\
G1403+05.80 & 10.592 & 9.992 & 9.870 & 82.97 & 0.025 \\
G1532-05.29 & 10.765 & 10.143 & 10.048 & 228.11 & 0.114 \\
G1448-05.103 & 10.707 & 9.989 & 9.895 & 171.28 & 0.063 \\
G1527+22.523 & 10.517 & 9.926 & 9.769 & -174.44 & 0.049 \\
G1358-16.167 & 10.236 & 9.625 & 9.498 & 93.13 & 0.085 \\
G1443-16.11 & 10.031 & 9.412 & 9.274 & 215.63 & 0.101 \\
G1955-22.448 & 10.288 & 9.509 & 9.385 & -206.40 & 0.129 \\
G2215-39.109 & 11.553 & 11.160 & 11.065 & -157.74 & 0.015 \\
G1934-50.224 & 10.224 & 9.755 & 9.651 & 222.28 & 0.063 \\
G2222-45.34 & 10.603 & 10.130 & 10.040 & 117.3 & NAN \\
G1851-39.1203 & 10.755 & 10.125 & 10.001 & -277.66 & 0.09 \\
G1942-44.358 & 10.599 & 9.932 & 9.754 & -297.42 & 0.051
\end{tabular}

Table 3: Current Study Star Information - Part 2

Dinescu (2002) searched for kinematic evidence of this in the solar neighborhood and found low-metallicity retrograde stars resembling that of $\omega$ Cen's orbit. High resolution spectroscopy was then observed of $12 \omega$ Cen candidates identified by Majewski et al. (2012) based on their $\omega$ Cen-like kinematics, and most of those were found by Majewski et al. to exhibit very high $[\mathrm{Ba} / \mathrm{Fe}]$ abundance patterns. This feature is thought to be unique to $\omega$ Cen stars with $[\mathrm{Fe} / \mathrm{H}] \gtrsim-1.7$.

The present paper expands on the Majewski et al 2012 work by exploiting high resolution spectroscopy data of 15 additional candidate $\omega$ Centauri stars. The candidate stars used for this study were chosen based on their being retrograde, with kinematics roughly consistent with the $\omega$ Cen tidal disruption model created by Majewski et al. (2012). These stars are shown in Table 2 and 3, which gives their ID, RA, Dec, kinematic data and magnitudes. Chemical tagging from these stars can provide more evidence that many stars in retrograde orbit around the Milky Way could have once been a part of $\omega$ Cen, as well as further constrain the model of $\omega$ Cen stripping by Majewski et al. (2000). Refining that model would further support the possibility of $\omega$ Centauri being a stripped dwarf spheroidal nucleus and clarify its history.

\section{NATURE OF THE DATA}

The origin of our sample can be traced back to Majewski et al. (2000), Majewski et al. (2004), Bizyaev et al. (2006), and Majewski et al. (2012). In 2000, the photometric Grid Giant Star Survey (GGSS) was conducted with 1302 evenly spaced fields across the sky. From this, Majewski et al. (2004) conducted a low-resolution $(R=2600)$ spectroscopic survey of 3,318 GGSS giants with the Swope LCO 1.0-m telescope. Through this Majewski et al. identified candidate retrograde stars near that plane also having kinematics consistent with being stripped debris from $\omega$ Cen, based on tidal destruction models of the system. Bizyaev et al. (2006) conducted a follow-up high-resolution spectroscopic survey of 774 stars using the McDonald 2.1 meter telescope, and Sandiford Echelle spectrograph with a resolution of $R=60,000$. Within the Bizyaev et al. sample was a subsample of a dozen of the Majewski et al. candidates, and 10 of the 12 were found by Majewski et al. to exhibit very high relative $\mathrm{Ba}$ abundances (as measured by the $\lambda=5854 \AA$ transition of Ba II) a peculiar characteristic of the $\omega$ Centauri system as originally shown by Smith et al. (2000) and Norris \& Costa (1995). Thus, these results showed the likelihood of a connection between these widely distributed field stars and $\omega$ Cen.

We expand on this previous work with another subsample of stars chosen from the same parent sample of GGSS stars with low-resolution spectra. For our subsample of 15 additional candidates, high-resolution spectra $(R=47,000)$ were obtained in the wavelength region (4140 - $6210 \AA)$ with the Ultraviolet Visual Echelle Spectrograph (UVES) on the 8.0$\mathrm{m}$ Very Large Telescope (VLT). The candidate stars used for this study were chosen based on their retrograde motion, and based on their kinematics being roughly consistent with the $\omega$ Cen debris in the tidal disruption model created by Majewski et al. (2012). This kinematic consistency can be seen in Figure 1, which overlays (in purple circles) the current sample of stars with Milky Way stars and those from $\omega$ Cen (in red and blue points). Most notably, the green shaded regions are those of the Majewski et al. model. It can be seen that the current sample of stars fit within this model. The sample stars were observed between the UT dates of May 7th, 2012 and July 30th, 2012 with exposure times between approximately 600 and 1300 seconds per star. The wavelength range was chosen due these redder wavelengths having a higher signal to noise ratio for our $\mathrm{K}$ giants. $\mathrm{K}$ giants were chosen for this observation for their brightness. The GGSS survey looked at $\mathrm{K}$ giants because their intrinsic brightness allows one to observe at greater distances.

\section{BARIUM ABUNDANCES}

Barium is abnormally abundant in $\omega$ Cen relative to other star systems known in the Milky Way, which makes that element effective for chemically tagging likely stripped members. This can be seen in Figure 2 in Majewski et al. (2012), also presented here as Figure 2. The $\omega$ Centauri stars clearly have higher barium abundances, at higher metallicities $([\mathrm{Fe} / \mathrm{H}] \gtrsim$ -1.7). Majewski et al. (2012) shows that their candidates stars follow this pattern, which strengthens the possibility of their relationship with $\omega$ Cen. 


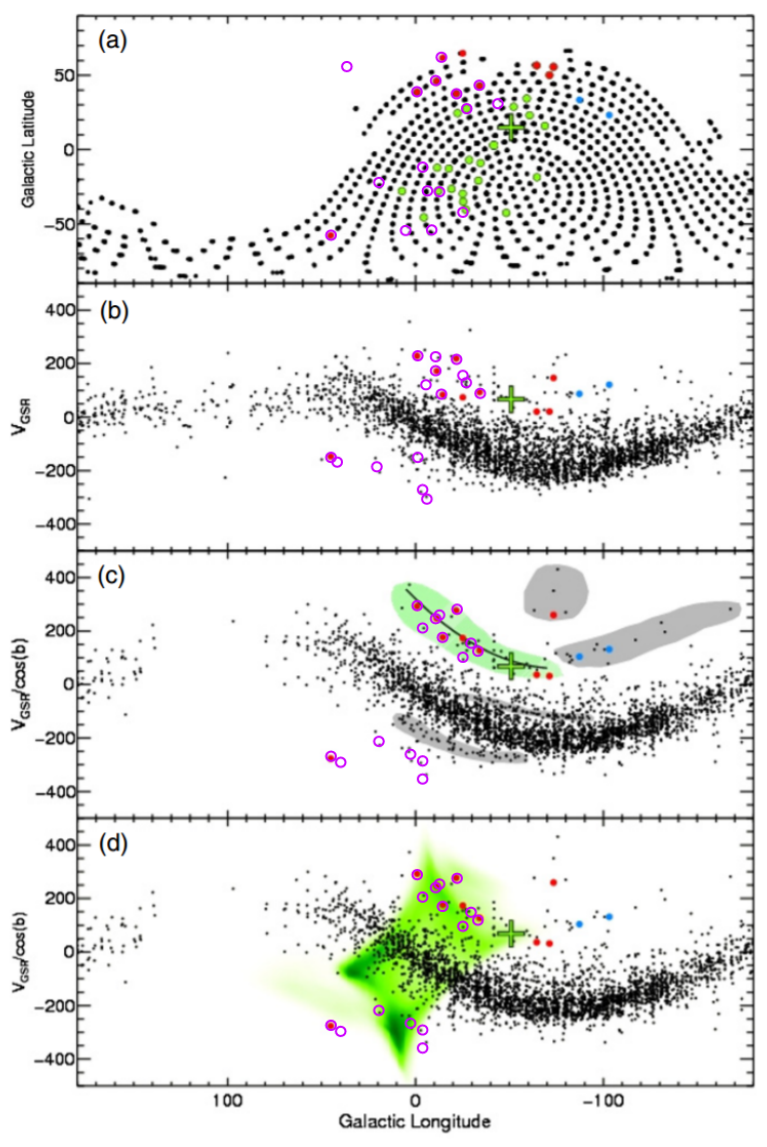

Figure 1. Distribution of the GGSS giant stars used in this study, originally plotted by Majewski et al. (2012) now with this study's data overlaid. Plots are in (a) Galactic Coordinates, (b) $v_{G S R}$ vs. longitude, and (c) $v_{b}=v_{G S R} / \cos b$ vs. longitude (for only $|b|<60 \mathrm{deg}$ in panel (c)). The curve and green shading in (c) highlight the arc of stars we believe contains $\omega$ Cen debris. Stars in this sequence are marked with green, red, or purple points in panel (a). Gray shadings show several other potential halo substructures. The position of the $\omega$ Cen core in all panels is shown by the large green cross. Panel (d) is the same as panel (c) but showing the "probability distribution" of $\omega$ Cen tidal debris based on our suite of models. Superposed red and blue points in all panels represent Majewski et al. (2012) stars with red designating those stars that follow the $\omega$ Cen $[\mathrm{Ba} / \mathrm{Fe}]-[\mathrm{Fe} / \mathrm{H}]$ patterns and blue those that do not. Purple circles represent stars from the current study.

Barium is an s-process element, typically formed by stars in the Asymptotic Giant Branch (AGB) phase. The overabundance of s-process elements in higher metallicity $\omega$ Cen stars is a reflection of the fact that there are multiple, highly diverse, stellar populations in $\omega$ Cen. Therefore $\omega$ Cen stars couldn't have all formed at roughly the same time, which is what happens in typical globular clusters, if non-AGB stars contain s-process elements.

The non-AGB stars that contain s-process elements must have formed from an interstellar medium (ISM) in $\omega$ Cen
The Astrophysical Journal LetTers, 747:L37 (6pp), 2012 March 10

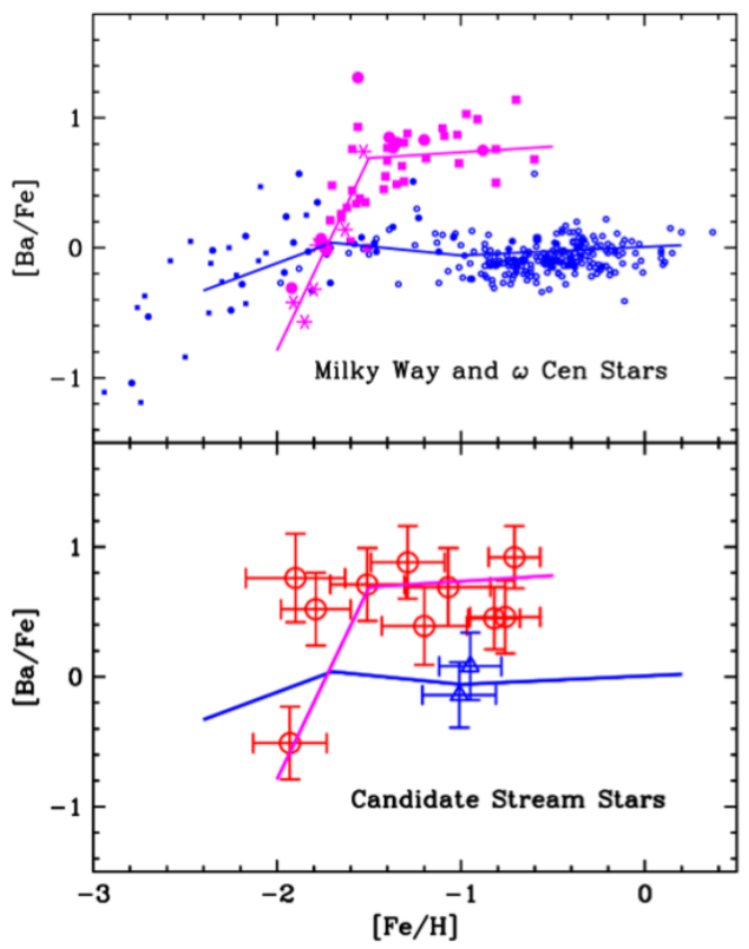

Figure 2. Figure 2 from Majewski et al. (2012). The top panel shows the $[\mathrm{Ba} / \mathrm{Fe}]$ vs $[\mathrm{Fe} / \mathrm{H}]$ distribution of Milky Way stars (blue points and line) and confirmed $\omega$ Cen stars (pink points and line). The Milky Way distribution is from Fulbright (2002), Johnson (2002), and Reddy et. al (2003, 2006). The $\omega$ Cen stars come from Francois et. al (1988), Norris and DaCosta (1995), and Smith et. al (200). The bottom panel shows the $\omega$ Cen candidate stars Majewski et. al (2012), with the red points indicating those that are likely stripped $\omega$ Cen stars based on their chemistry, and blue points indicating those that are likely Milky Way stars.

which already included debris from an AGB star that had the time to live a full life and die, releasing its s-process elements into the ISM. Given that AGB stars are cool and have a longer life cycle, this means that the populations in $\omega$ Cen must be separated by longer periods of time. Given that the populations in globular clusters are very uniform, s-process elements are typically not seen in these systems. S-process elements are, however, seen in dwarf spheroidal systems, and their presence provides further evidence to $\omega$ Cen being a stripped dwarf spheroidal nucleus.

\section{METHODOLOGY}

\subsection{Data Reduction}

In the stellar spectra for our sample, the VLT pipeline had performed the wavelength calibration and merged the orders into one FITS file spanning the entire range of wavelengths observed (4140-6200 ̊). Unfortunately, these orders were not merged in an optimal way and left intensity "bumps" of 
low intensity to the highest and lowest wavelengths of each order, and high intensity corresponding to the center of each order. We thus first cut out data that did not contain these strange artifacts, and continuum-normalized the usable data using the Image Reduction Analysis Facility (IRAF.)

The elements measured were $\mathrm{Ba}$ II, Fe I and II, Mg, and $\mathrm{Na}$. The NIST atomic spectral database, provides key atomic data such as the center, $\log (\mathrm{gf})$, and excitation potential for lines from each element. We chose those lines that fit within the spectral regions free of the strange artifacts mentioned above and, in the case of Fe I where there were several lines to choose from, had quality ratings above a D+ in the NIST spectral database (see Table 4).

\subsection{Equivalent Widths Measurements}

For the lines of interest, the equivalent widths were measured using iSpec, an open-source framework for spectral analysis (Blanco-Cuaresma et. al 2014). To determine the accuracy of these equivalent widths measurements, we compared them to 1.) equivalent width data measured by hand, 2.) a Python script using Sherpa packages, and 3.) a Python script using PyEW. Of these four methods, iSpec and the equivalent widths measured by hand were the most consistent with each other, and iSpec was the only automatic method that did not contain occasional measurements far off from the other three measurements. As it was by far the most reliable automated method, we measured the entire group of stars using iSpec.

\subsection{Model Atmospheres and Abundances}

Using our measured equivalent widths, we created model atmospheres for each star. First the effective temperature of each star was calculated using the infrared flux method (IRFM) outlined by Hernandez \& Bonifacio (2009) using the $J, H, K$, metallicity, reddening, and surface gravity. This compared theoretical fluxes obtained from ATLAS models, and compared them directly with the observed 2MASS J,H,K magnitudes. The surface gravity was assumed to be $\log (g)=$ 2.5 , as appropriate for a typical giant star. The initial guess for metallicity was adopted as $[\mathrm{Fe} / \mathrm{H}]=-1.5$, using the McMasterHarris Cluster Catalog for $\omega$ Centauri. Because the stars in our sample are all in low reddening regions, and because these values are the initial guess in an iterative process via iSpec, the reddening from Schlegel et al. (1998) is sufficient for our purposes.

We then input these initial guesses into iSpec's model atmosphere parameter calculation. iSpec calculates model atmospheres automatically using the MOOG driver. It (1) performs an ionization balance: the average Fe I and Fe II abundances are made equal (2) ensures no trends are found when abundances are plotted against excitation potential, and (3) ensures no trends are found when abundances are plotted against equivalent width.

\begin{tabular}{ccccc} 
Element & $\lambda$ Center $(\AA)$ & EP $(\mathrm{EV})$ & $\log (\mathrm{gf})$ & Quality \\
\hline \hline $\mathrm{Na}$ & 5889.951 & 0.000 & 0.108 & $\mathrm{AA}$ \\
$\mathrm{Na}$ & 5895.924 & 0.000 & -0.194 & $\mathrm{AA}$ \\
$\mathrm{Na}$ & 6154.225 & 2.102 & -1.547 & $\mathrm{~A}$ \\
$\mathrm{Na}$ & 6160.740 & 2.104 & -1.246 & $\mathrm{~A}$ \\
$\mathrm{Mg}$ & 4571.096 & 0.000 & -5.623 & $\mathrm{D}$ \\
$\mathrm{Mg}$ & 4702.991 & 4.346 & -0.440 & $\mathrm{~B}+$ \\
$\mathrm{Mg}$ & 5528.405 & 4.346 & -0.498 & $\mathrm{~B}+$ \\
$\mathrm{Mg}$ & 5711.088 & 4.346 & -1.724 & $\mathrm{~B}$ \\
$\mathrm{Fe}$ & 4514.184 & 3.047 & -2.050 & $\mathrm{D}+$ \\
$\mathrm{Fe}$ & 4517.524 & 3.071 & -1.858 & $\mathrm{C}+$ \\
$\mathrm{Fe}$ & 4547.847 & 3.547 & -1.012 & $\mathrm{D}+$ \\
$\mathrm{Fe}$ & 4566.515 & 3.301 & -2.376 & $\mathrm{D}+$ \\
$\mathrm{Fe}$ & 4587.127 & 3.573 & -1.737 & $\mathrm{C}$ \\
$\mathrm{Fe}$ & 4602.001 & 1.608 & -3.134 & $\mathrm{~A}$ \\
$\mathrm{Fe}$ & 5816.373 & 4.548 & -0.601 & $\mathrm{~B}$ \\
$\mathrm{Fe}$ & 5855.076 & 4.607 & -1.478 & $\mathrm{~B}$ \\
$\mathrm{Fe}$ & 5856.088 & 4.294 & -1.327 & $\mathrm{C}$ \\
$\mathrm{Fe}$ & 6120.249 & 0.915 & -5.970 & $\mathrm{~B}+$ \\
$\mathrm{Fe}$ & 6127.906 & 4.143 & -1.399 & $\mathrm{D}+$ \\
$\mathrm{Fe}$ & 6136.615 & 2.453 & -1.402 & $\mathrm{~B}$ \\
$\mathrm{Fe}$ & 6137.691 & 2.588 & -1.402 & $\mathrm{~B}$ \\
$\mathrm{Fe}$ & 6151.617 & 2.176 & -3.295 & $\mathrm{~B}$ \\
$\mathrm{Fe}$ & 6165.360 & 4.143 & -1.473 & $\mathrm{C}+$ \\
$\mathrm{Fe} \mathrm{II}$ & 6173.334 & 2.223 & -2.880 & $\mathrm{~B}$ \\
$\mathrm{Fe}$ & 6180.203 & 2.728 & -2.591 & $\mathrm{D}+$ \\
$\mathrm{Fe} \mathrm{II}$ & 4520.221 & 2.807 & -2.600 & $\mathrm{D}$ \\
$\mathrm{Fe}$ & 4555.887 & 2.828 & -2.160 & $\mathrm{C}$ \\
$\mathrm{Fe}$ & 4576.328 & 2.844 & -2.920 & $\mathrm{C}$ \\
$\mathrm{Fe}$ & 61492.835 & 2.844 & -3.090 & $\mathrm{C}$ \\
$\mathrm{Fe}$ & 3.889 & -2.841 & $\mathrm{E}$ \\
$\mathrm{Fe}$ & 0.000 & 0.140 & $\mathrm{~B}$ \\
$\mathrm{Fe}$ & 0.604 & -0.908 & $\mathrm{~B}$ \\
$\mathrm{Fe}$ & & -0.032 & $\mathrm{~B}$
\end{tabular}

Table 4: Atomic lines used for abundances. These were found using the NIST atomic spectral database, and the Fe I lines have quality values above a D+ in NIST. 
The parameters that best converge on these three features in iSpec are found to be the parameters for the model atmosphere of that stars. This method was tested against finding the model atmospheres by hand using MOOGs abfind driver, and the two methods were found to be very consistent with one another.

We then interpolated the data from the MARCS model atmosphere Plez (2008) database to create our model atmospheres. The values and errors of these model atmospheric parameters can be seen in Tables 5 and 6. Then, with MOOG's abfind routine, we were able to use these model atmospheres to estimate abundances for the $\mathrm{Mg}$ and $\mathrm{Na}$ elements.

\begin{tabular}{ccccc} 
Star ID & $\mathrm{T}(\mathrm{K})$ & $\log (\mathrm{g})$ & {$[\mathrm{Fe} / \mathrm{H}]$} & $v_{\text {micro }}(\mathrm{km} / \mathrm{s})$ \\
\hline \hline $\mathrm{G} 1488-28.133$ & 4407 & 0.87 & -1.57 & 1.80 \\
$\mathrm{G} 2109-61.187$ & 4672 & 1.75 & -1.35 & 1.76 \\
$\mathrm{G} 2237-16.2017$ & 4422 & 0.490 & -1.75 & 1.94 \\
$\mathrm{G} 1403+05.80$ & 4600 & 2.12 & -0.71 & 1.84 \\
$\mathrm{G} 1532-05.29$ & 5037 & 2.55 & -1.32 & 1.65 \\
$\mathrm{G} 1448-05.103$ & 4399 & 1.32 & -1.15 & 1.95 \\
$\mathrm{G} 1527+22.523$ & 4590 & 2.47 & -0.02 & 2.01 \\
$\mathrm{G} 1358-16.167$ & 6220 & 4.90 & -0.32 & 2.65 \\
$\mathrm{G} 1443-16.11$ & 4582 & 1.38 & -1.89 & 1.74 \\
$1955-22.448$ & 4228 & 0.340 & -1.56 & 2.37 \\
$\mathrm{G} 2215-39.109$ & 5818 & 3.70 & -0.66 & 0.750 \\
$\mathrm{G} 1934-50.224$ & 5194 & 2.78 & -1.28 & 1.18 \\
$\mathrm{G} 2222-45.34$ & 5251 & 3.42 & -0.69 & 2.54 \\
$1851-39.1203$ & 4390 & 0.100 & -1.56 & 1.31 \\
$1942-44.358$ & 4679 & 2.30 & -0.61 & 2.23
\end{tabular}

Table 5: Model Atmosphere Data (From iSpec)

Due to Barium having several isotopes and hyperfine structure, MOOGs abfind driver was not sufficient to measure the abundances. The abfind driver does not account for this hyperfine or isotopic structure, however MOOGs synth driver does. The synth driver creates a synthetic spectrum which is then manually fit to the data. We used MOOG, our model atmospheres, and detailed line lists of transitions around our barium lines of interest from Kurucz line lists to create synthetic spectra of the barium lines we aimed to measure. Using MOOG's synth feature, we then fit these synthetic lines to obtain our barium abundances. An example of this type of fit can be seen in Figure 3. These fits were done manually, and in this particular fit the yellow line is chosen, corresponding to $[\mathrm{Ba} / \mathrm{Fe}]=-0.5$. Typical fits were within a $\chi^{2}<0.03$.

\section{RESULTS}

\begin{tabular}{ccccc} 
Star ID & $\Delta \mathrm{T}(\mathrm{K})$ & $\Delta \log (\mathrm{g})$ & $\Delta[\mathrm{Fe} / \mathrm{H}]$ & $\Delta v_{\text {micro }}(\mathrm{km} / \mathrm{s})$ \\
\hline \hline $\mathrm{G} 1488-28.133$ & 6.3 & 0.23 & 0.03 & 0.05 \\
$\mathrm{G} 2109-61.187$ & 7.2 & 0.17 & 0.02 & 0.02 \\
G2237-16.2017 & 20.4 & 0.17 & 0.03 & 0.04 \\
$\mathrm{G} 1403+05.80$ & 26.3 & 0.15 & 0.05 & 0.04 \\
$\mathrm{G} 1532-05.29$ & 60.7 & 0.26 & 0.03 & 0.09 \\
$\mathrm{G} 1448-05.103$ & 10.4 & 0.22 & 0.04 & 0.04 \\
$\mathrm{G} 1527+22.523$ & 25.8 & 0.08 & 0.04 & 0.12 \\
$\mathrm{G} 1358-16.167$ & 241.0 & 0.21 & 0.10 & 0.32 \\
$\mathrm{G} 1443-16.11$ & 41.4 & 0.21 & 0.07 & 0.11 \\
$1955-22.448$ & 21.5 & 0.23 & 0.06 & 0.07 \\
$\mathrm{G} 2215-39.109$ & 67.3 & 0.08 & 0.04 & 0.19 \\
$\mathrm{G} 1934-50.224$ & 55.6 & 0.18 & 0.03 & 0.10 \\
$\mathrm{G} 2222-45.34$ & 13.5 & 0.14 & 0.02 & 0.21 \\
$1851-39.1203$ & 58.3 & 0.36 & 0.04 & 0.10 \\
$1942-44.358$ & 35.5 & 0.15 & 0.04 & 0.06
\end{tabular}

Table 6: Model Atmosphere Error Data (From iSpec) for values in Table 2. The $[\mathrm{Fe} / \mathrm{H}]$ was calculated from the standard deviation of $[\mathrm{Fe} / \mathrm{H}]$ given by iSpec, while the other parameter values were calculated by fixing and alternating all parameters but one $\left(T_{e f f} \pm 100 \mathrm{~K}, \log (g) \pm 0.1\right.$, and $\left.v_{\text {micro }} \pm 0.1\right)$ and observing the changes of the non-fixed parameter.

Figure 4 shows the distribution of $[\mathrm{Ba} / \mathrm{Fe}]-[\mathrm{Fe} / \mathrm{H}]$ for Milky Way stars (shown in gray) and for $\omega$ Centauri stars (shown in purple), overlaid with the stripped $\omega$ Cen candidates identified by Majewski et al. (2012) (in red) and all the stars from the current sample (in green). One can see that approximately twelve of the fifteen stars studied here follow the $\omega \mathrm{Cen}[\mathrm{Ba} / \mathrm{Fe}]-[\mathrm{Fe} / \mathrm{H}]$ trend, with only three appearing to better follow that of Milky Way stars. However, for $-2<[\mathrm{Fe} / \mathrm{H}]<-1.5$ the $[\mathrm{Ba} / \mathrm{Fe}]$ of $\omega$ Cen stars and Milky Way stars overlap, so it is hard to definitively say whether all of these stars were once a part of $\omega$ Cen. To confirm this, we would need ancillary information, such as the kinematical proper motion of these stars, to show that they also fit into the tidal disruption model from Majewski et al. (2012). Nevertheless, these results are still quite promising as many of the higher metallicity stars exhibit barium abundances well above what would be expected from Milky Way stars, and our sample overall has chemistry that is strongly consistent with that of $\omega$ Cen. Tables 7 and 8 also show the exact values for the abundances calculated with their errors.

According to Norris \& Costa (1995), the $[\mathrm{Mg} / \mathrm{Fe}]$ and $[\mathrm{Na} / \mathrm{Fe}]$ abundance ranges of $\omega$ Cen are -0.2 to 0.6 and -0.5 to 1.2, respectively. The $[\mathrm{Mg} / \mathrm{Fe}]$ and $[\mathrm{Na} / \mathrm{Fe}]$ abundances of the sample (Figures 5,6) are consistent with the ranges expected 


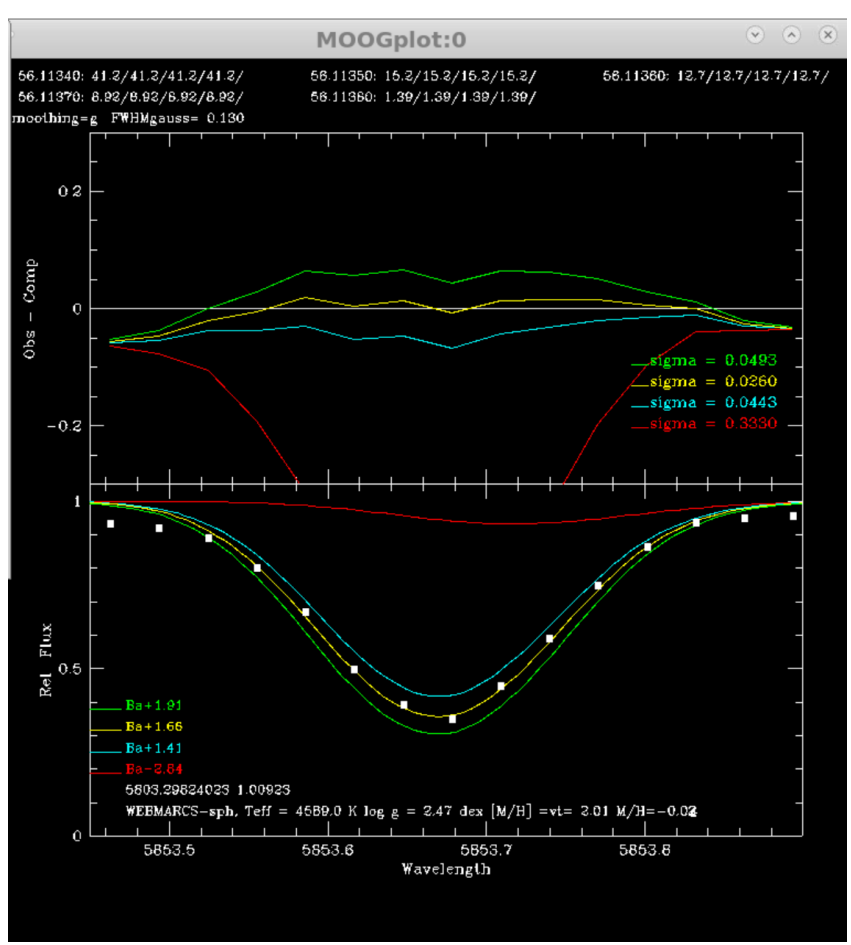

Figure 3. Example of 5853.673 Barium II line fit in MOOGs synth driver for star $\mathrm{G} 1527+22.523$. This was done manually, with the yellow line being the one chosen, translating to $[\mathrm{Ba} / \mathrm{Fe}]=-0.5$ for this measurement.

\begin{tabular}{ccccc} 
Star ID & {$[\mathrm{Fe} / \mathrm{H}]$} & {$[\mathrm{Ba} / \mathrm{Fe}]$} & {$[\mathrm{Mg} / \mathrm{Fe}]$} & {$[\mathrm{Na} / \mathrm{Fe}]$} \\
\hline \hline G1448-28.133 & -1.57 & 0.05 & 0.44 & -0.05 \\
G2109-61.187 & -1.35 & 0.22 & 0.30 & 0.01 \\
G2237-16.2017 & -1.75 & -0.20 & 0.11 & -0.36 \\
G1403+05.80 & -0.71 & 1.20 & 0.30 & 0.12 \\
G1532-05.29 & -1.32 & 0.33 & 0.05 & -0.22 \\
G1448-05.103 & -1.15 & 0.32 & 0.36 & -0.05 \\
G1527+22.523 & -0.02 & -0.40 & 0.10 & 0.34 \\
G1358-16.167 & -0.32 & 0.90 & -0.26 & -0.16 \\
G1443-16.11 & -1.89 & -0.15 & 0.40 & 0.49 \\
$1955-22.448$ & -1.56 & -0.20 & 0.37 & 0.07 \\
G2215-39.109 & -0.66 & 0.43 & -0.20 & -0.20 \\
G1934-50.224 & -1.28 & 0.33 & 0.20 & 0.09 \\
G2222-45.34 & -0.69 & 0.02 & -0.15 & -0.10 \\
$1851-39.1203$ & -1.56 & 0.43 & 0.87 & 0.41 \\
$1942-44.358$ & -0.61 & -0.12 & NAN & NAN
\end{tabular}

Table 7: Abundance Data

from a star stripped from $\omega$ Cen. However, the results are too preliminary, particularly without aluminum abundances which would show $\mathrm{Mg}-\mathrm{Al}$ anti-correlation and $\mathrm{Na}-\mathrm{Al}$ correlation trends expected from all globular clusters, but also

\begin{tabular}{ccccc} 
Star ID & $\Delta[\mathrm{Fe} / \mathrm{H}]$ & $\Delta[\mathrm{Ba} / \mathrm{Fe}]$ & $\Delta[\mathrm{Mg} / \mathrm{Fe}]$ & $\Delta[\mathrm{Na} / \mathrm{Fe}]$ \\
\hline \hline G1448-28.133 & 0.03 & 0.15 & 0.08 & 0.06 \\
$\mathrm{G} 2109-61.187$ & 0.02 & 0.03 & 0.04 & 0.06 \\
$\mathrm{G} 2237-16.2017$ & 0.03 & 0.10 & 0.24 & 0.11 \\
$\mathrm{G} 1403+05.80$ & 0.05 & 0.06 & 0.15 & 0.09 \\
$\mathrm{G} 1448-05.103$ & 0.04 & 0.12 & 0.02 & 0.08 \\
$\mathrm{G} 1527+22.523$ & 0.04 & 0.15 & 0.23 & 0.15 \\
$\mathrm{G} 1358-16.167$ & 0.10 & 0.01 & 0.70 & 0.41 \\
$\mathrm{G} 1443-16.11$ & 0.07 & 0.15 & 0.03 & 0.04 \\
$1955-22.448$ & 0.06 & 0.01 & 0.03 & 0.09 \\
$\mathrm{G} 2215-39.109$ & 0.04 & 0.03 & 0.26 & 0.37 \\
$\mathrm{G} 1934-50.224$ & 0.03 & 0.12 & 0.11 & 0.05 \\
$\mathrm{G} 2222-45.34$ & 0.02 & 0.06 & 0.12 & 0.13 \\
$1851-39.1203$ & 0.04 & 0.03 & 0.22 & 0.01 \\
$1942-44.358$ & 0.04 & 0.07 & NAN & NAN
\end{tabular}

Table 8: Standard Error calculated for abundance values from Table $7-[\mathrm{Fe} / \mathrm{H}]$ was calculated from the standard deviation given by iSpec, while the other abundance errors were calculated by the standard deviation calculated from the difference between measurements in multiple line transitions.

exhibited by stars in $\omega$ Cen. Combined with the abnormally high $[\mathrm{Ba} / \mathrm{Fe}]$ values, a feature not seen in ordinary globular clusters, these correlations would further point to the stars having been stripped from $\omega$ Cen. Additionally, the sodium abundances for all stars appear to match those of solar levels.

\section{CONCLUSION}

We observed fifteen stars using high-resolution spectroscopy with the purpose of chemically determining whether or not these stars are stripped $\omega$ Cen candidates as suggested by their unusual kinematics.

The fact that the majority of these stars show chemical evidence of once being a part of $\omega$ Cen provides evidence that many retrograde stars in the particular kinematical region predicted by the model were in fact once a part of $\omega$ Cen. Finally, these results provide further evidence towards $\omega$ Cen being a stripped dwarf spheroidal nucleus, and not a globular cluster.

Future work to investigate this hypothesis could include obtaining the proper motions and abundances of additional elements, such as aluminum and oxygen, to further prove the connection between these candidate stars and $\omega$ Cen. These elements could be used to observe the Mg-Al, Na-O anticorrelations and $\mathrm{Na}-\mathrm{Al}$ correlations seen in $\omega$ Cen as well as in globular clusters. Observing these correlative and anticorrelative behaviors would involve taking new samples of the data with a greater range of wavelengths. However, With the spectra we currently have, we can measure and test the 
- Milky Way Stars (Fulbright 2000, Johnson 2002, and Reddy et al $(2003,2006))$

- Omega Centauri Stars (Francois et al 1988, Norris \& Da Costa 1995, and Smith et al 2000)

\ Majewski et al 2012

- Current Data

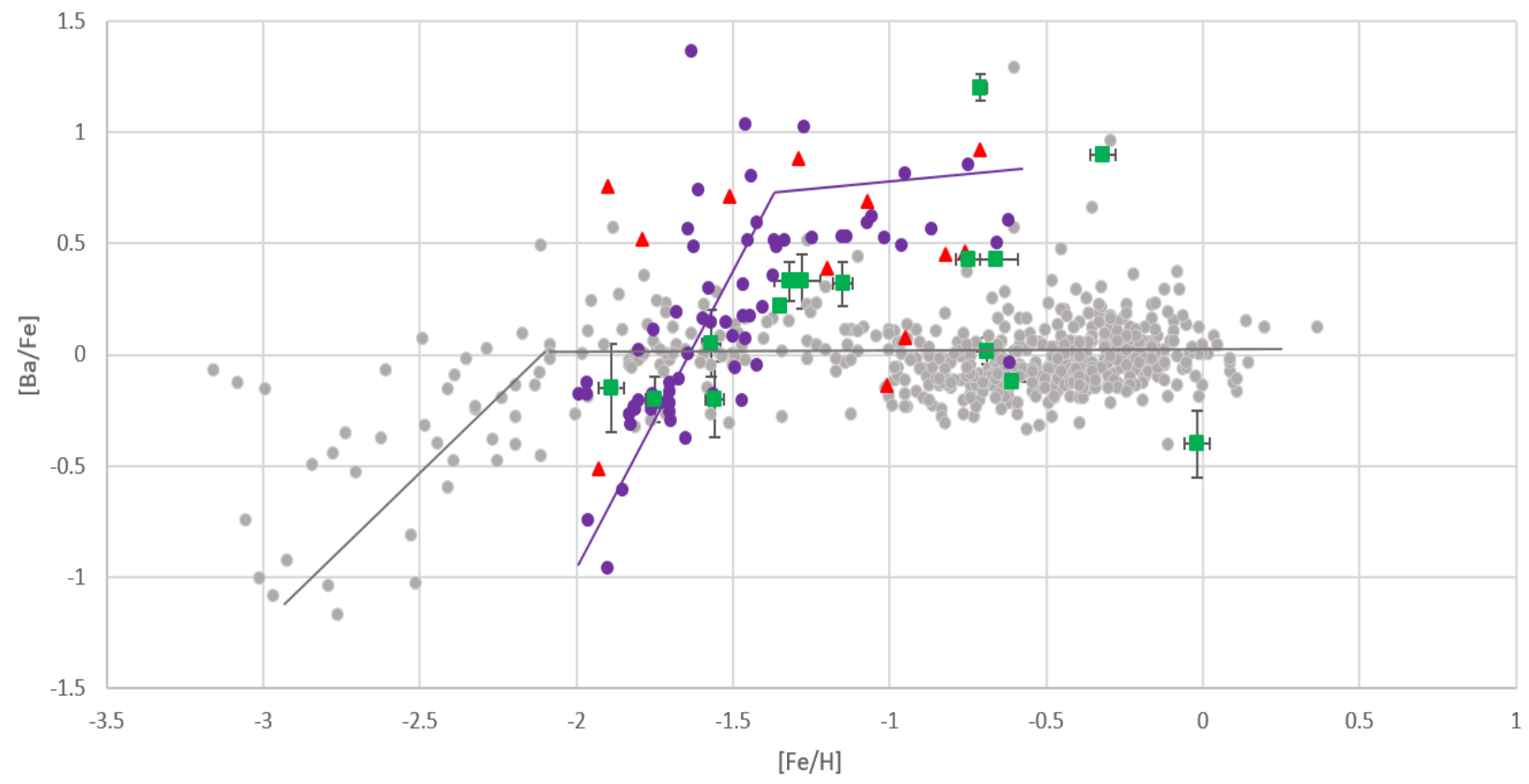

Figure 4. The distribution of $[\mathrm{Ba} / \mathrm{Fe}]-[\mathrm{Fe} / \mathrm{H}]$ for Milky Way stars (gray points and line), overlaid with the same for stars in the $\omega$ Cen system (purple points and line). The Milky Way star data come from Fulbright (2000), Johnson (2002), Reddy et al. (2003), and Reddy et al. (2006), while the $\omega$ Cen data come from Francois et al. (1988), Norris \& Costa (1995), and Smith et al. (2000). Additionally, data from Majewski et al. (2012) is shown in red. The results for stars studied here are shown in green. This barium trend in $\omega$ Cen comes from enrichment from early generations of AGB stars, which produce s-process elements such as barium. These processes presumed to have happened to $\omega$ Cen do not occur in normal globular clusters.

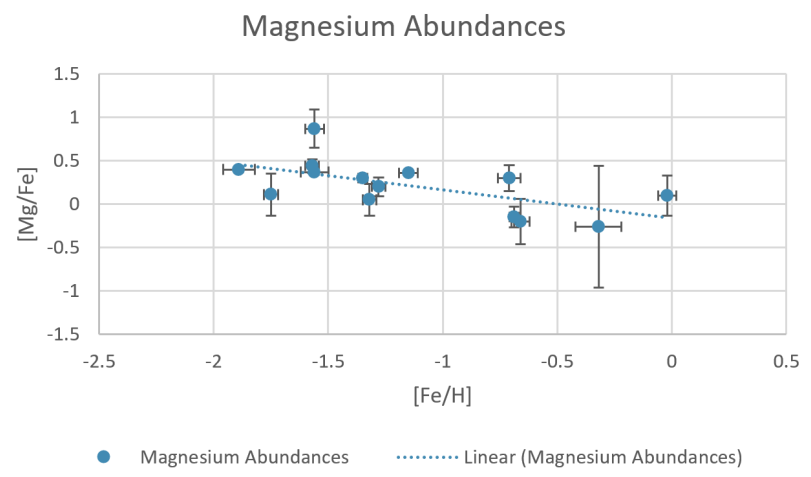

Figure 5. A graph of $[\mathrm{Mg} / \mathrm{H}]-[\mathrm{Fe} / \mathrm{H}]$, which shows a slight downward trend as metallicity increases.

C-N anti-correlation. Additionally, even more stars could be observed through these same methods.

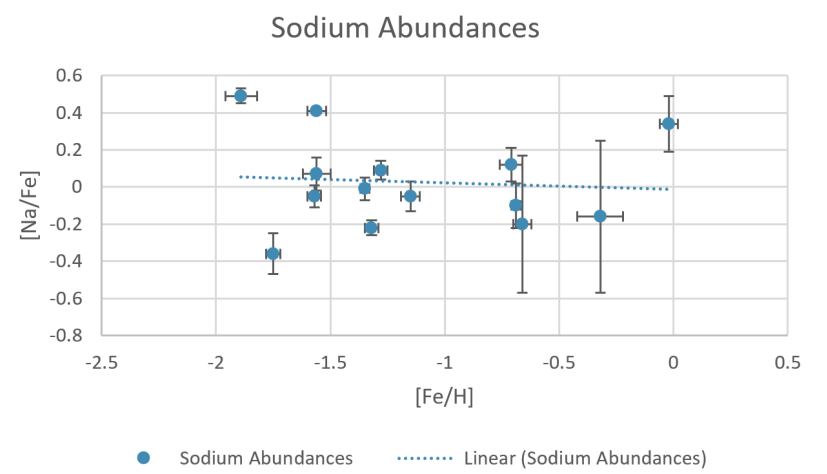

Figure 6. A graph of $[\mathrm{Mg} / \mathrm{H}]-[\mathrm{Fe} / \mathrm{H}]$, which shows no clear trend and is centered around zero, indicating that the Sodium abundances of these stars are roughly all solar.

This work was largely supported by Jennifer Sobeck, Steven Majewski, Christian Hayes, and David Nidever. 


\section{REFERENCES}

????

Asplund, M., Grevesse, N., Sauval, A. J., \& Scott, P. 2009, ApJ

Bender, R., Burstein, D., \& Faber, S. M. 1992, ApJ

Bizyaev, D., Smith, V. V., Arenas, J., et al. 2006, ApJ

Blanco-Cuaresma, S., Soubiran, C., Heiter, U., \& Jofre, P. 2014, ApJ

Carretta, E., Bragaglia, A., Gratton, R. G., et al. 2010, ApJ

de Ven, G. V., den Bosch, R. V., Verolme, E., \& de Zeeuw, P. 2006, ApJ

Denissenkov, P. A., Costa, G. S. D., Norris, J. E., \& Weiss, A. 1998, ApJ

Dinescu, D. 2002, ApJ

Dyck, H., Belle, G. V., \& Thompson, R. R. 1998, ApJ

Fellhauer, M., Evans, N. W., Belokurov, V., Wilkinson, M. I., \& Gilmore, G. 2007, ApJ

Fellhauer, M., Belokurov, V., Evans, N. W., et al. 2006, ApJ

Francois, P., Spite, M., \& Spite, F. 1988, ApJ

Fulbright, J. P. 2000, ApJ

Harris, W. E. 1996, ApJ

-. 2010, ApJ

Hernandez, J. I. G., \& Bonifacio, P. 2009, ApJ

Hilker, M., Kayser, A., Richtler, T., \& Willemson, P. 2004, ApJ

Hubble, E., \& Humason, M. L. 1931, ApJ, 74, 43

Jalali, B. 2011, PhD thesis,

AA(Ludwig-Maximilians-UniversitÃd't MÃijnchen), 2011
Johnson, J. A. 2002, ApJ

Kruijssen, D., \& Mieske, S. ????

Law, D., \& Majewski, S. 2016, ApJ

Lee, Y. W., Joo, J. M., Sohn, Y. J., et al. 1999, ApJ

Majewski, S. R., Nidever, D. L., Smith, V. V., et al. 2012, ApJ

Majewski, S. R., Patterson, R. J., Dinescu, D. I., et al. 2000, ApJ

Majewski, S. R., Kunkel, W. E., Law, D. R., et al. 2004, ApJ

Mateo, M. 1998, ApJ

Mizutani, A., Masashi, C., \& Sakamoto, T. 2003, ApJ

Norris, J. E., \& Costa, G. S. D. 1995, ApJ

Odenkirchen, M., Grebel, E. K., Dehnen, W., et al. 2002, ApJ

Plez, B. 2008, ApJ

Pryor, C., \& Meylan, G. 1993, ApJ

Reddy, B. E., Lambert, D. L., \& Prieto, C. A. 2006, MNRAS

Reddy, B. E., Tomkin, J., Lambert, D. L., \& Prieto, C. A. 2003, MNRAS

Ricardo, R., Majewski, S. R., Carlin, J. L., et al. 2008, ApJ

Roos, M. 2012, ApJ

Schlegel, D. J., Finkbeiner, D. P., \& Davis, M. 1998, ApJ

Smith, V. V., Suntzeff, N. B., Cunha, K., et al. 2000, ApJ

Sohn, S. T., Majewski, S. R., Munoz, R. R., et al. 2007, ApJ

Tailo, M., Criscienzo, M. D., D’Antona, F., Caloi, V., \& Ventura, P. 2016, ApJ

Ven, G. V., Bosch, R. V., Verolme, E., \& Zeeuw, P. D. 2008, Leiden, The Netherlands: Astronomy and Astrophysics 